\title{
Taurocholate Transport by Rat Intestinal Basolateral Membrane Vesicles Evidence for the Presence of an Anion Exchange Transport System
}

\author{
Sharon L. Weinberg, * Gerhard Burckhardt, $\ddagger$ and Frederick A. Wilson* \\ *Department of Medicine, Milton S. Hershey Medical Center, Pennsylvania State University, Hershey, Pennsylvania 17033; and \\ $\ddagger$ Max-Planck-Institut für Biophysik, Frankfurt am Main, Federal Republic of Germany
}

\begin{abstract}
The transport of bile acid was studied in basolateral membrane vesicles isolated from rat small intestine. Taurocholate transport into an osmotically reactive intravesicular space was $\mathrm{Na}^{+}$independent. The uptake of taurocholate in jejunal and ileal vesicles preloaded with sulfate was stimulated with respect to uptake in unpreloaded vesicles. Glycocholate inhibited the transstimulation of taurocholate uptake by sulfate. Sulfate and taurocholate uptake in ileal vesicles preloaded with bicarbonate was stimulated with respect to uptake in unpreloaded vesicles. Taurocholate inhibited the transstimulation of sulfate uptake by bicarbonate. When ileal vesicles were loaded with p-aminohippurate, an early transstimulation of taurocholate was found that exceeded equilibrium uptake, was insensitive to a $\mathrm{K}^{+}$diffusion potential, and was cis-inhibited by taurocholate, glycocholate, pyruvate, $p$ aminohippurate, probenecid, chloride, sulfate, and bicarbonate. These data indicate the presence of an anion exchanger in intestinal basolateral membrane vesicles that may be involved in the exit of bile acids from the enterocyte.
\end{abstract}

\section{Introduction}

From previous in vivo studies in man (1) and laboratory animals (2) and in vitro studies with intestinal segments (2-6), isolated epithelial cells $(7,8)$, and brush border membrane vesicles (9-12), it is recognized that bile acids are transported across the small intestinal mucosal membrane either by ionic or nonionic passive diffusion and, in addition, across the ileal brush border membrane by an active $\mathrm{Na}^{+}$-dependent transport process. The energy to drive the $\mathrm{Na}^{+}$-bile acid transporter that enables the bile acid to accumulate within the cell against its electrochemical gradient is derived from a $\mathrm{Na}^{+}$gradient across the brush-border membrane. The $\mathrm{Na}^{+}$gradient arises from the extrusion of $\mathrm{Na}^{+}$ from the cell. The hydrolysis of ATP by $\mathrm{Na}^{+}-\mathrm{K}^{+}$-ATPase located on the basolateral membrane provides the energy for this $\mathrm{Na}^{+}$$\mathrm{K}^{+}$exchange. Thus, bile acid uptake in the ileum may be defined as a secondary active transport (13).

In contrast to our knowledge of events involved in the uptake of bile acids by the luminal membrane, relatively little is known regarding their exit across the basolateral membrane of the in-

This work was presented in part in 1984 at the VIII International Bile Acid Meeting, Falk Symposium No. 42, in Berne, Switzerland.

Address reprint requests to Dr. Wilson, Division of Gastroenterology, Milton S. Hershey Medical Center, Box 850, Hershey, PA 17033.

Received for publication 22 October 1984 and in revised form 18 March 1986.

J. Clin. Invest.

(C) The American Society for Clinical Investigation, Inc. 0021-9738/86/07/0044/07 \$1.00

Volume 78, July 1986, 44-50 testinal epithelial cell. It is possible that the flow of bile acid from the cell across the basolateral membrane is directed "downhill" from a region of higher concentration to one of lower concentration, despite a lower free concentration due to potential bile acid binding or to incorporation within intracellular components. Furthermore, the movement of bile acid anions out of the cell is favored by the negative intracellular potential (13). Thus, the transport of bile acid across the basolateral membrane may not require the input of energy or specialized mechanisms that are necessary to transport bile acids across the brush-border membrane. However, there is evidence that sugars are transported across intestinal basolateral membrane vesicles by sodium-independent facilitated diffusion (14). A more recent report with renal basolateral membrane vesicles suggests that a common exchanger for inorganic and organic anions may be involved in reabsorption and secretion in the proximal tubule of the rat kidney (15). At physiological $\mathrm{pH}$, bile acids are in the ionized state with a single negative charge on the side chain (16). Therefore, the possibility that such an anion exchange system exists for bile acids in intestinal basolateral membrane vesicles was explored in the present studies.

\section{Methods}

Membrane preparation. Basolateral membrane vesicles were prepared from rat small intestine based on centrifugation in a Percoll medium (17). In brief, male Sprague-Dawley rats weighing 180-200 g were killed by a blow on the neck and bled. The small intestine was removed and divided into ten equal segments. Segments 2-3 and 8-9 were used for studies of jejunum and ileum, respectively. The segments were flushed with ice-cold lactated Ringer's solution and then filled with warmed $\left(37^{\circ} \mathrm{C}\right.$ ) buffer (buffer II) containing $1.5 \mathrm{mM} \mathrm{KCl}, 96 \mathrm{mM} \mathrm{NaCl}, 8 \mathrm{mM}$ $\mathrm{KH}_{2} \mathrm{PO}_{4}, 5.6 \mathrm{mM} \mathrm{Na} \mathrm{HPO}_{4}, 27 \mathrm{mM} \mathrm{Na}$ citrate, and $2 \mathrm{mM}$ dithiothreitol, pH 7.4. The segments were clamped with hemostats and incubated in buffer II without dithiothreitol (buffer III) in a shaking bath at $37^{\circ} \mathrm{C}$ for $15 \mathrm{~min}$. The segments were unclamped, and the buffer and mucus were removed by pulling the segments between the fingers. The segments were refilled with ice-cold buffer (buffer IV) containing 250 $\mathrm{mM}$ mannitol and $12 \mathrm{mM}$ Hepes-Tris, $\mathrm{pH}$ 7.4. On an ice-cold plate, the segments were palpated for $2 \mathrm{~min}$ to release cells from mucosa. The cells were drained into polystyrene centrifuge tubes and brought up to 250 $\mathrm{ml}$ in buffer IV. The cells were centrifuged at $200 \mathrm{~g}$ for $5 \mathrm{~min}$. The cell pellet was homogenized in $250 \mathrm{ml}$ buffer IV for $3 \mathrm{~min}$ in an Omni-mixer at maximal speed. The homogenate was brought up to $300 \mathrm{ml}$ with buffer IV and centrifuged at $2,500 \mathrm{~g}$ for $20 \mathrm{~min}$. The supernatant was collected and centrifuged at $22,000 \mathrm{~g}$ for $25 \mathrm{~min}$. After the supernatant was discarded, the fluffy layer of the pellet was resuspended in $90 \mathrm{ml}$ of buffer IV and homogenized in a glass/teflon homogenizer (20 strokes, $1,200 \mathrm{rev} / \mathrm{min})$. Then $95.47 \mathrm{~g}$ of homogenate was added to $13.67 \mathrm{~g}$ of Percoll (12.5\%) and mixed by everting. The mixture was centrifuged at $48,000 \mathrm{~g}$ for $45 \mathrm{~min}$. The band of basolateral membranes was removed and diluted 1:4 vol/vol with buffer (buffer $\mathrm{V}$ ) containing $100 \mathrm{mM}$ mannitol, $100 \mathrm{mM} \mathrm{KCl}$, and $12 \mathrm{mM}$ Hepes-Tris, pH 7.4. The diluted membranes were centrifuged at $48,000 \mathrm{~g}$ for $20 \mathrm{~min}$. The pellet was resuspended three times in appropriate preincubation buffer for transport studies and centrifuged at $48,000 \mathrm{~g}$ for $20 \mathrm{~min}$. The final vesicle pellet 
was collected in a tuberculin syringe and suspended in the desired volume of preincubation buffer. The composition of the preincubation buffers varied from experiment to experiment and is therefore provided in the legends to the figures.

Enzyme and protein determinations. The purity of the basolateral membranes was assessed by determination of the activity of marker enzymes. Enzyme and protein determinations were carried out on unfrozen samples kept on ice within $5 \mathrm{~h}$ of the rats being killed. $\mathrm{Na}^{+}-\mathrm{K}^{+}-\mathrm{ATPase}$ (E.C. 3.6.1.3) activity was measured as described by Scharschmidt et al. (18). Leucine aminopeptidase (E.C. 3.4.11.1) activity was measured using a Kit 124869 (Boehringer Mannheim Biochemicals, Indianapolis, IN). NAD oxidoreductase (E.C. 1.6.99.2) was measured in the presence of $0.3 \mathrm{mM} \mathrm{KCN}$ as described by Sottocasa et al. (19). Acid phosphatase (E.C. 3.1.3.2) was measured using a Kit 104-AS (Sigma Chemical Co., St. Louis, MO). Succinate-cytochrome $c$ oxidoreductase (E.C. 1.3.99.1) activity was measured according to the method of Fleischer and Fleischer (20). Protein was determined by the method of Lowry et al. (21) after precipitation with ice-cold $10 \%$ trichloroacetic acid using bovine serum albumin as a standard.

The specific activity of $\mathrm{Na}^{+}-\mathrm{K}^{+}$-ATPase, the marker enzyme for basolateral membranes, was enriched 18.3 \pm 1.4-fold (mean $\pm \mathrm{SE}$ of eight determinations) with respect to starting homogenate. On the other hand the specific activity of the leucine aminopeptidase, a marker for brush border membranes, was negatively enriched at $0.3 \pm 0.4$-fold (mean $\pm \mathrm{SE}$, $n=8$ ). Thus, the basolateral membranes were purified over 60 -fold with respect to the brush borders in the initial homogenate. Moreover, the specific activities of KCN-resistant NAD oxidoreductase and acid phosphatase were not enriched, such that the basolateral membranes were purified 230- and 278-fold with respect to microsomes and lysosomes, respectively. Finally, the activity of succinate-cytochrome $c$ oxidoreductase, a marker for mitochondria, was undetectable in the basolateral membrane preparation.

Transport measurements. Uptake of radiolabeled substrate was determined by the membrane filtration technique of Hopfer et al. (22). Typically, the transport reaction was initiated by adding $20 \mu \mathrm{l}$ of preincubated vesicles to $180 \mu \mathrm{l}$ of incubation buffer kept in a water bath at $37^{\circ} \mathrm{C}$. The composition of the incubation media is given in the legends to the figures. At desired time intervals, 40 - or $80-\mu \mathrm{l}$ aliquots were removed from the incubation suspension and diluted in $1 \mathrm{ml}$ of ice-cold stop solution that had the same composition as the incubation medium except for the isotope. The stop solution containing the vesicles was immediately pipetted onto the middle of a prewashed, prechilled filter (cellulose nitrate, $0.45-\mu \mathrm{m}$ pore size, Sartorius Filters, Inc., Hayward, CA) kept under suction. The filter was immediately rinsed with $5 \mathrm{ml}$ of ice-cold stop solution and then solubilized in Brays solution. The radioactivity remaining on the filter was counted with standard liquid scintillation techniques. After correction of medium radioactivity bound to the filters in the absence of vesicle membranes, absolute solute uptake was calculated and is expressed as picomoles per milligram protein. All experiments were performed in triplicate with freshly prepared membranes on two separate days.
Materials. Nonlabeled bile acids were obtained from Steraloids, Inc. (Wilton, NH) and Calbiochem-Behring Corp. (San Diego, CA). $\left[{ }^{3} \mathrm{H}(\mathrm{G})\right]$ Taurocholic acid $(6.8 \mathrm{Ci} / \mathrm{mmol}),\left[{ }^{14} \mathrm{C}\right] \mathrm{D}$-glucose $(57.1 \mathrm{mCi} / \mathrm{mmol})$ and $\left[{ }^{35} \mathrm{~S}\right]$ sulfate $(5 \mathrm{mCi} / \mathrm{ml})$ were obtained from New England Nuclear (Boston, MA). The radiopurity of the labeled taurocholate acid was found to be $>95 \%$ using thin-layer chromatography. All reagents were of analytical grade.

Statistical analysis. Values were expressed as mean \pm SE of triplicate determinations from two separate membrane preparations. Means of the groups were compared by unpaired $t$ test. $P$ values $<0.05$ were considered significant.

\section{Results}

Taurocholate metabolism, binding, and transport. Initial experiments were performed to discriminate possible membrane metabolic effects from those associated with binding or transport. Ileal basolateral membrane vesicles were incubated up to 60 min with radiolabeled taurocholate. Vesicular radioactivity was extracted overnight with methanol and submitted to thin-layer chromatography using the solvent system propionic acid/isoamyl alcohol/l-propanol/ $\mathrm{H}_{2} \mathrm{O}$ (60:60:40:30, by volume). Greater than $95 \%$ of the radioactivity co-chromatographed with the taurocholate standard, indicating lack of metabolism of the bile acid by the membrane preparation.

The osmotic sensitivity of taurocholate uptake by the vesicle membrane preparation was determined in order to distinguish between membrane binding and transport. Intravesicular volume may be modified by the addition of varying concentrations of impermeant solute to the incubation medium. Under these circumstances, varying taurocholate uptake into an internal space directly reflects changes in intravesicular volume at equilibrium. Fig. 1 shows the effect of increasing osmolarity of the incubation medium on taurocholate and D-glucose equilibrium uptake (at $60 \mathrm{~min}$ ) by ileal basolateral membrane vesicles. An increase in the osmotic gradient across the membrane from 143 to 500 mosM cellobiose decreased D-glucose uptake by $127 \%$, whereas taurocholate uptake changed by $49 \%$, indicating that only a relatively small portion of taurocholate was taken up into an osmotically reactive intravesicular space. Indeed, extrapolation of the linear relationship in Fig. 1 yielded a positive Y-intercept that suggests significant membrane binding at infinite osmolarity of the incubation medium. Moreover, ileal vesicles were loaded with radiolabeled taurocholate and subsequently diluted and washed in ice-cold stop solution containing 0 or $1 \mathrm{mM}$ unlabeled taurocholate. The presence of $1 \mathrm{mM}$ taurocholate decreased the bile acid uptake by $15 \%$ when compared with uptake in the

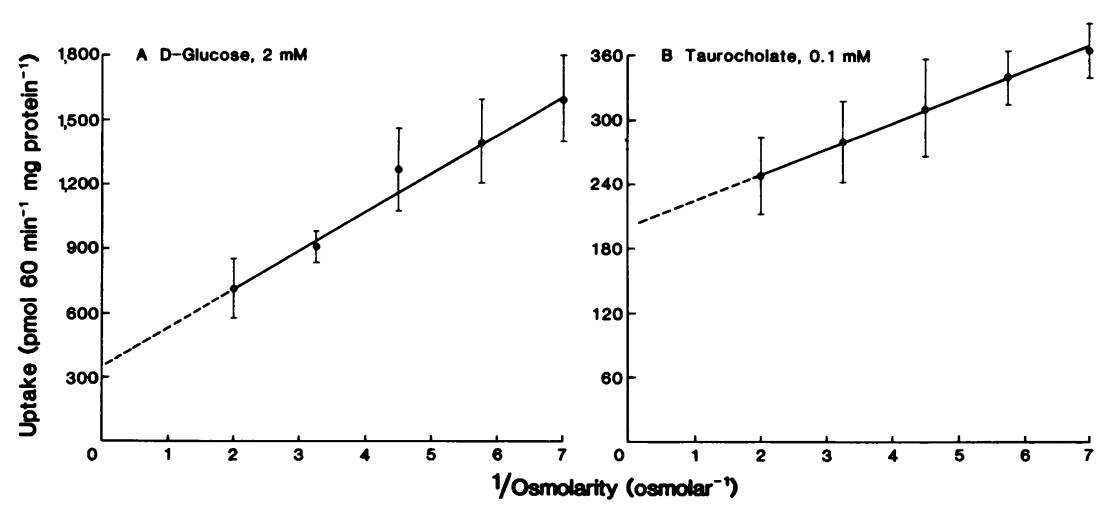

Figure 1. Effect of medium osmolarity on the 60min uptake of D-glucose and taurocholate. Ileal basolateral membrane vesicles were preloaded with $250 \mathrm{mM}$ mannitol, $0.2 \mathrm{mM} \mathrm{CaCl}_{2}$, and $30 \mathrm{mM}$ Hepes-Tris buffer, $\mathrm{pH} 7.4$, containing $2 \mathrm{mM}$ $\left[{ }^{14} \mathrm{C}\right]$ D-glucose $(A), 0.1 \mathrm{mM}\left[{ }^{3} \mathrm{H}\right]$ taurocholate $(B)$, and varying concentrations of cellobiose to yield the final osmolarity in the incubation media as plotted on the abscissa. Each point represents the mean $\pm \mathrm{SE}$ for two runs of triplicate determinations. 
absence of the bile acid. This observation (data not shown) suggests that $\sim 85 \%$ of taurocholate partitions into the membrane interior, binds to the internal surface of vesicles, and/or is taken up into the intravesicular space where it cannot be easily displaced $(23,24)$.

Previous studies indicated that the bile acid uptake by ileal brush border membrane vesicles is sodium dependent (9-12). In contrast, as shown in Fig. 2, taurocholate uptake by ileal basolateral membrane vesicles in the presence of a sodium gradient (outside greater than inside) was similar to uptake in a potassium gradient. The finding suggests not only a lack of sodium dependency for bile acid uptake by basolateral membrane vesicles but also a lack of contamination of this membrane preparation by brush-border membranes.

Evidence for the presence of an anion exchange transport system for bile acid. The presence of an anion exchange transporter in renal and intestinal basolateral membrane vesicles has been demonstrated using radiolabeled sulfate as a substrate (15, $25,26)$. A characteristic of this transporter is that it can be driven additionally by a transmembrane $\mathrm{pH}$ difference (15). Studies to determine the influence of a proton gradient on bile acid uptake are subject to artifact because the binding of bile acid to membrane surfaces is sensitive to changes in $\mathrm{pH}$ (27). For these reasons, sulfate was chosen as a test anion to determine whether the anion exchange transporter in intestinal basolateral membrane vesicles is sensitive to a transmembrane $\mathrm{pH}$ difference and is shared by taurocholate. The effect of an imposed $\mathrm{pH}$ gradient on $0.1 \mathrm{mM}$ radiolabeled sulfate uptake by jejunal and ileal vesicles is illustrated in Fig. 3, $A$ and $B$, respectively. When membrane vesicles were preincubated in pH 8.4 buffer and then exposed to incubation buffer of $\mathrm{pH} \mathrm{6.4,} \mathrm{a} \mathrm{transient} \mathrm{intravesicular}$ accumulation (overshoot) of sulfate was noted that attained a level three times greater than the eventual equilibrium value at $60 \mathrm{~min}$. When the uncoupler, carbonyl cyanide $p$ trifluoromethoxyphenylhydrazone (FCCP), ${ }^{1}$ was added to the media, the level of intravesicular sulfate uptake significantly declined. These results suggest that the imposition of a transmembrane $\mathrm{pH}$ difference stimulates the transient accumulation of sulfate against its concentration gradient. With dissipation of the $\mathrm{pH}$ difference by FCCP, the driving force for uphill anion transport is removed and the level of intravesicular sulfate accumulation is decreased. The stimulation of sulfate uptake by a transmembrane $\mathrm{pH}$ difference could be driven by a proton diffusion potential. In as much as experiments were performed in the presence of the ionophore valinomycin and equimolar extra- and intravesicular potassium concentrations this possibility seems unlikely (15). Fig. 3 also shows that the addition of 0.5 $\mathrm{mM}$ taurocholate to the media inhibited proton gradient-driven sulfate uptake. This inhibition could occur via competition for the same carrier.

To explore the possibility that sulfate and bile acids share a common anion exchange transport system, experiments to demonstrate the transstimulation of radiolabeled taurocholate uptake by sulfate were performed with basolateral membrane vesicles. As shown in Fig., $4 A$ and $B$, when jejunal and ileal vesicles were prepared in buffer containing $25 \mathrm{mM}$ sulfate and incubated in a sulfate-free, equiosmolar medium, initial (20 s) taurocholate uptake was stimulated almost twofold compared

1. Abbreviations used in this paper: FCCP, p-trifluoromethoxyphenylhydrazone; $\mathrm{PAH}, p$-aminohippurate.

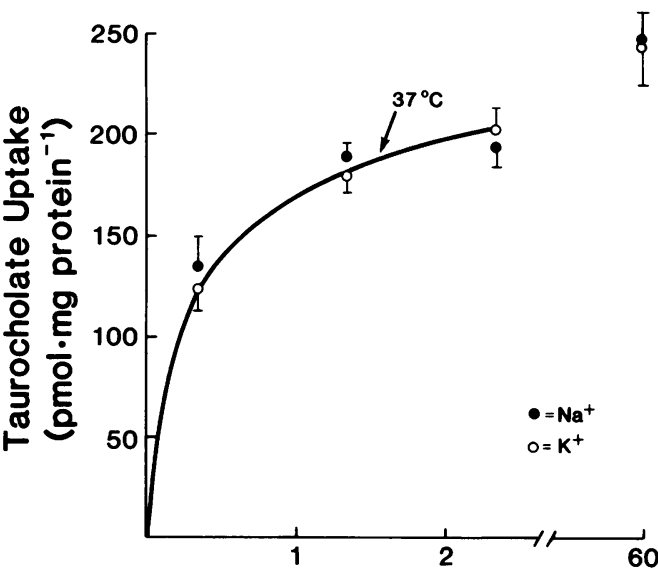

Time of Incubation ( $\mathrm{min}$ )

Figure 2. Lack of effect of a sodium gradient on taurocholate uptake by rat basolateral membrane vesicles. Ileal vesicles were preloaded with $250 \mathrm{mM}$ mannitol, $0.2 \mathrm{mM} \mathrm{CaCl}_{2}, 10 \mathrm{mM} \mathrm{MgCl}_{2}, 100 \mathrm{mM}$ $\mathrm{KCl}$, and $10 \mathrm{mM}$ Hepes-Tris buffer, $\mathrm{pH}$ 7.4. Vesicles then were incubated at $37^{\circ} \mathrm{C}$ in the same medium containing $0.1 \mathrm{mM}$

$\left[{ }^{3} \mathrm{H}\right]$ taurocholate $(\mathrm{O})$ or in medium where $100 \mathrm{mM} \mathrm{KCl}$ was replaced by $100 \mathrm{mM} \mathrm{NaCl}(\bullet)$. Each point represents the mean $\pm \mathrm{SE}$ for two runs of triplicate determinations.

with its uptake in the total absence of sulfate. This difference persisted up to $2 \mathrm{~min}$ of incubation. At equilibrium (60 min), the uptake of taurocholate in the presence of sulfate was not significantly different from the uptake without sulfate. Furthermore, from the data in Fig. 4, it is apparent that the initial uptake of taurocholate exceeded the uptake at $60 \mathrm{~min}$, suggesting a transient intravesicular accumulation of taurocholate. The uptake in the absence of sulfate did not exceed significantly the value at equilibrium. Fig. 4 also shows that $0.5 \mathrm{mM}$ glycocholate in the incubation medium significantly inhibited the sulfate-
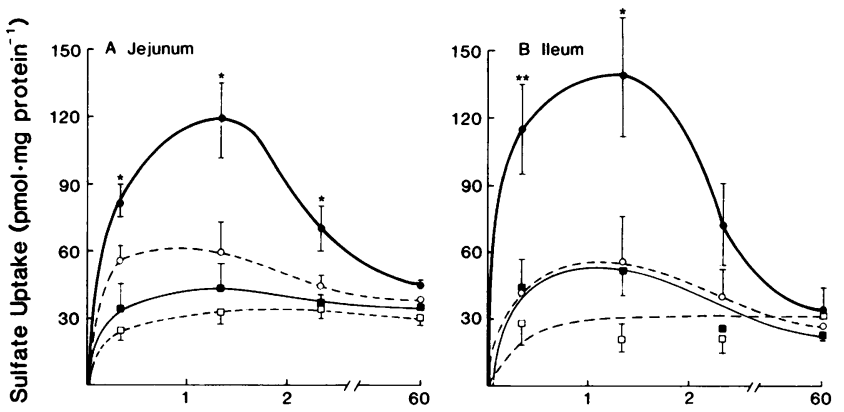

Time of Incubation ( $\mathrm{min}$ )

Figure 3. Effect of a proton gradient on sulfate uptake by rat jejunal $(A)$ and ileal $(B)$ basolateral membrane vesicles. Vesicles were preloaded with $150 \mathrm{mM}$ tetramethylammonium, $50 \mathrm{mM}$ potassium, 200 $\mathrm{mM}$ gluconate, $62.5 \mu \mathrm{g} / \mathrm{ml}$ valinomycin, and $50 \mathrm{mM} \mathrm{N,N-bis-(2-hy-}$ droxyethyl) glycine (Bicin) titrated with tetramethylammonium hydroxide to $\mathrm{pH}$ 8.4. Then uptake of $0.1 \mathrm{mM}$ radiolabeled sulfate was assayed in the same medium containing $50 \mathrm{mM} N$-morpholinoethanesulfonic acid (MES) titrated to pH 6.4 instead of Bicin. $5 \mu \mathrm{M}$ carbonyl cyanide FCCP and $0.5 \mathrm{mM}$ taurocholate (TC) were present as shown in the figure. Each point represents the mean \pm SE for two runs of triplicate determinations. *Equals $P<0.05$, and ***equals $P<0.01$ for sulfate uptake ( $0 \mathrm{mM}$ TC; $0 \mu \mathrm{M}$ FCCP) vs. other values. $-\bullet-\mathrm{TC}$, $0 \mathrm{mM}$; FCCP, $0 \mu \mathrm{M}$; - - ० - TC, $0.5 \mathrm{mM}$; FCCP, $0 \mu \mathrm{M}$; - TC, $0 \mathrm{mM}$; FCCP, $5 \mu \mathrm{M}$; - - - - TC, $0.5 \mathrm{mM}$; FCCP, $5 \mu \mathrm{M}$. 


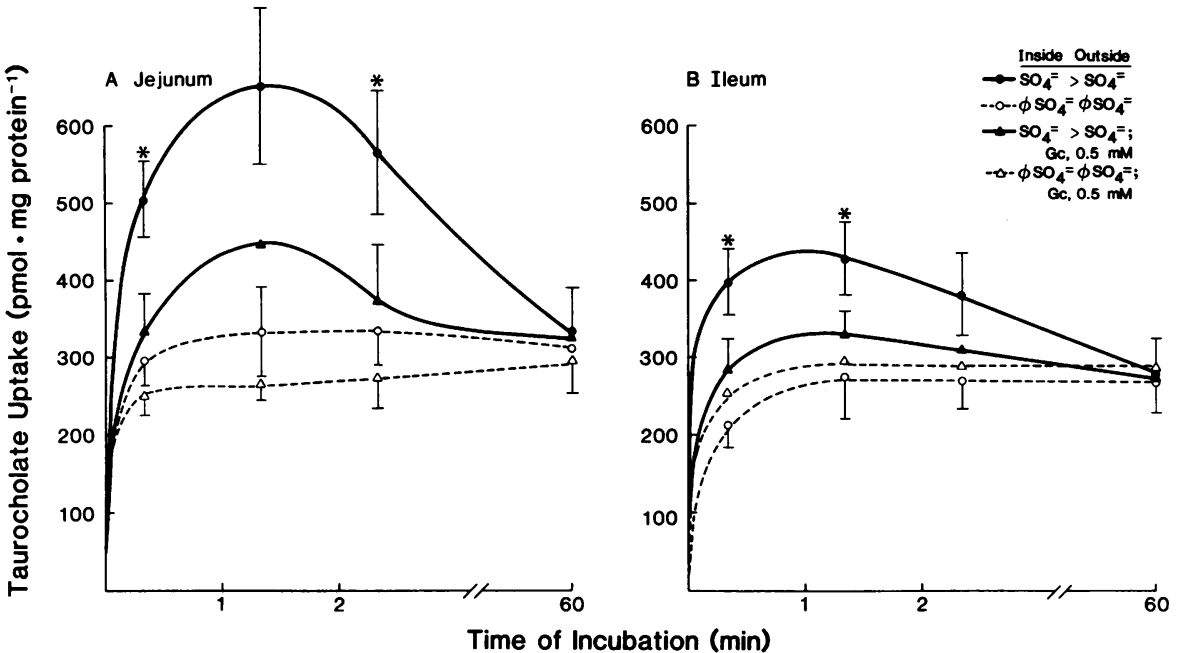

Figure 4. Effect of sulfate transstimulation on taurocholate uptake by rat jejunal $(A)$ and ileal $(B)$ basolateral membrane vesicles. Vesicles were preloaded with $150 \mathrm{mM}$ tetramethylammonium, $50 \mathrm{mM}$ potassium, 200 $\mathrm{mM}$ gluconate, $12 \mathrm{mM}$ Hepes-Tris buffer, $\mathrm{pH} 7.4$, and $0(\mathrm{O}, \Delta)$ or $25 \mathrm{mM}$ sulfate and $175 \mathrm{mM}$ gluconate $(\bullet, \Delta)$, and then uptake of $0.1 \mathrm{mM}\left[{ }^{3} \mathrm{H}\right]$ taurocholate was assayed in the sulfate-free medium containing in addition $0(\bullet, 0)$ or $0.5 \mathrm{mM}(\Delta, \Delta)$ glycocholate (Gc). All experiments were performed in the presence of valinomycin and FCCP. Each point represents the mean \pm SE for two runs of triplicate determinations. *Equals $P$ $<0.05$ for taurocholate uptake $\left(\mathrm{SO}_{4}>\mathrm{SO}_{4}\right.$; $0 \mathrm{mM} \mathrm{Gc}$ ) vs. other values. transstimulated transport of $0.1 \mathrm{mM}$ taurocholate but not the sulfate-independent uptake. Since the experiment was performed in the presence of FCCP, valinomycin, and equimolar extraand intravesicular potassium concentrations, it is likely that stimulated taurocholate uptake represents bile acid-sulfate exchange rather than indirect coupling to $\mathrm{pH}$ and/or electrical potential differences.

The ability of bicarbonate to transstimulate the transport of both sulfate and taurocholate was next examined. Ileal basolateral membrane vesicles were preincubated in buffer containing the presence or absence of $25 \mathrm{mM}$ bicarbonate, $\mathrm{pH}$ 7.4. The two membrane fractions then were exposed to radiolabeled $0.1 \mathrm{mM}$ sulfate or $0.1 \mathrm{mM}$ taurocholate in bicarbonate-free equiosmolar buffer, $\mathrm{pH}$ 7.4. The presence of an in to out bicarbonate gradient not only resulted in enhanced sulfate (Fig. $5 \mathrm{~A}$ ) and taurocholate (Fig. $5 B$ ) uptake by membrane vesicles with respect to their uptake in the total absence of bicarbonate, but also led to their transient intravesicular accumulation above equilibrium uptake. The uptake in the absence of bicarbonate did not exceed significantly the value at equilibrium. At equilibrium, the uptake of sulfate and taurocholate in the presence of bicarbonate was similar to uptake in the absence of bicarbonate. Fig. $5 A$ also shows that the addition of $0.1 \mathrm{mM}$ taurocholate to the incubation medium inhibited bicarbonate-transstimulated sulfate uptake but not the bicarbonate-free uptake. In separate experiments (data not shown), the presence of FCCP in the medium did not affect bicarbonate-transstimulated uptake. Therefore, sulfate and taurocholate uptake appears to occur via bicarbonate anion exchange rather than via indirect coupling to a proton gradient resulting from bicarbonate efflux.

To determine whether transstimulation of bile acid by another organic anion is possible, studies were carried out with $p$ aminohippurate (PAH). As shown in Fig. 6, when vesicles were loaded with $10 \mathrm{mM}$ PAH and then diluted 20-fold into medium containing radiolabeled $0.1 \mathrm{mM}$ taurocholate, the uptake of the bile acid at $20 \mathrm{~s}$ was stimulated over twofold with respect to uptake in the absence of PAH. The externally directed PAH gradient was able to drive taurocholate accumulation above equilibrium uptake. Moreover, the transstimulation of taurocholate by PAH could be blocked by the organic anion probenecid. In the absence of PAH-transstimulating conditions, probenecid did not inhibit significantly taurocholate uptake. The ionophores, FCCP and valinomycin, and equal potassium concentration again were added to the media to minimize indirect effects on transport that arise from transmembrane $\mathrm{pH}$ and potential differences generated by test anions.

The next experiment was carried out to determine whether the transstimulation of taurocholate by PAH is sensitive to a
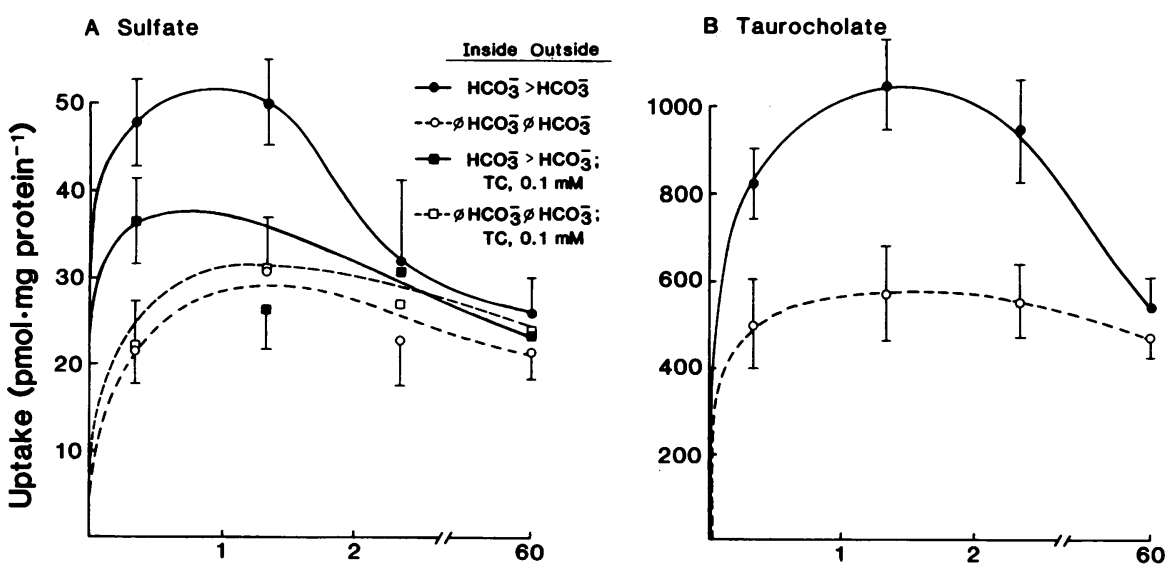

Time of Incubation (min)
Figure 5. Effect of bicarbonate transstimulation on sulfate and taurocholate uptake by basolateral membranes. Ileal vesicles were preloaded with $150 \mathrm{mM}$ tetramethylammonium, $50 \mathrm{mM}$ potassium, $200 \mathrm{mM}$ gluconate, $12 \mathrm{mM}$ Hepes-Tris buffer, and 0 $(O, \square)$ or $25 \mathrm{mM}$ bicarbonate and $175 \mathrm{mM}$ gluconate $(\bullet, \bullet), \mathrm{pH} 7.4$, and then uptake of $0.1 \mathrm{mM}$ radiolabeled sulfate $(A)$ or taurocholate $(B)$ was assayed in the bicarbonatefree medium, $\mathrm{pH}$ 7.4. All experiments were performed in the presence of valinomycin. Sulfate uptake experiments were performed in the absence $(\bullet, 0)$ or the presence $(\bullet, \square)$ of $0.1 \mathrm{mM}$ unlabeled taurocholate (TC). Each point represents the mean $\pm S E$ for two runs of triplicate determinations. 


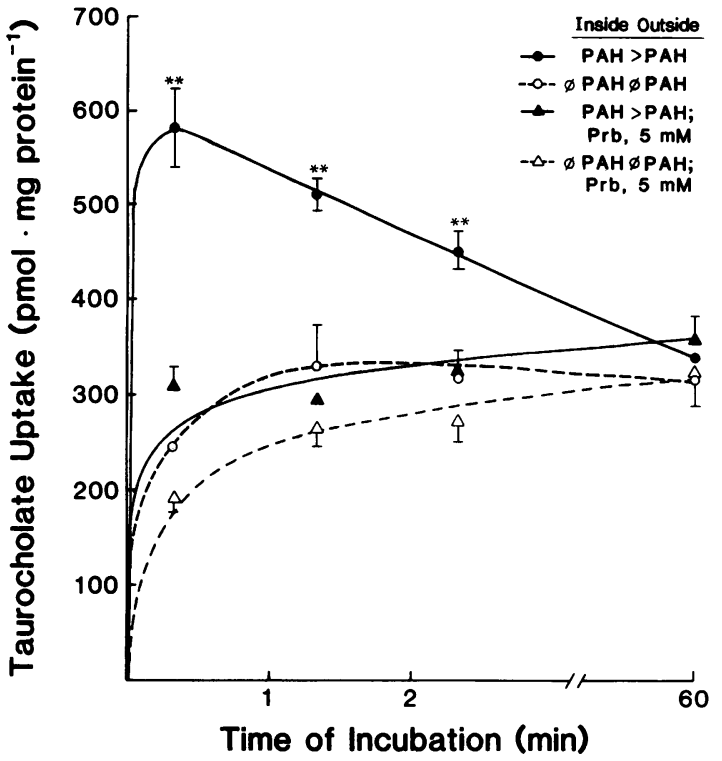

Figure 6. Effect of PAH transstimulation on taurocholate uptake by basolateral membrane vesicles. Ileal vesicles were preloaded with 150 $\mathrm{mM}$ tetramethylammonium, $50 \mathrm{mM}$ potassium, $200 \mathrm{mM}$ gluconate,

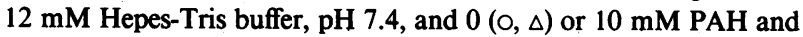
$190 \mathrm{mM}$ gluconate $(\bullet, \Delta)$, and then uptake of $0.1 \mathrm{mM}\left[{ }^{3} \mathrm{H}\right]$ taurocholate was assayed in the same medium containing $0.5 \mathrm{mM}$ PAH and 0 $(\bullet, 0)$ or $5 \mathrm{mM}(\Delta, \Delta)$ probenecid (Prb). All experiments were performed in the presence of valinomycin and FCCP. Each point represents the mean $\pm S E$ for two runs of triplicate determinations. ${ }^{* *}$ Equals $P<0.01$ for PAH transstimulated uptake vs. other values.

membrane potential. Ileal basolateral membrane vesicles were preloaded in the presence or absence of $10 \mathrm{mM}$ PAH and then incubated in the presence of $50 \mathrm{mM}$ potassium (potassium outside greater than potassium inside) with or without valinomycin. In the presence of valinomycin, the rapid influx of extravesicular potassium generates an electrochemical potential with the intravesicular space relatively negative. As shown in Table I, the imposition of a potassium diffusion potential had no significant effect $(P>0.05)$ on taurocholate uptake at $20 \mathrm{~s}$ and $60 \mathrm{~min}$

Table I. Lack of Effect of a Valinomycin-induced Membrane Potential on Taurocholate Uptake

\begin{tabular}{lll}
\hline & \multicolumn{2}{l}{ Time of incubation } \\
\cline { 3 - 3 } Conditions* & $20 \mathrm{~s}$ & $60 \mathrm{~min}$ \\
\hline PAH: Transstimulation & & \\
$\quad$ With valinomycin & $699.6 \pm 24.0 \ddagger$ & $359.6 \pm 28.7$ \\
$\quad$ Without valinomycin & $674.3 \pm 43.9$ & $357.7 \pm 23.1$ \\
No PAH: Transstimulation & & \\
$\quad$ With valinomycin & $317.6 \pm 17.8$ & $339.3 \pm 18.8$ \\
Without valinomycin & $339.6 \pm 19.9$ & $341.7 \pm 16.1$ \\
\hline
\end{tabular}

* Ileal vesicles were preloaded with potassium-free, $12 \mathrm{mM}$ Hepes-Tris buffer, pH 7.4, in the presence or absence of $10 \mathrm{mM} \mathrm{PAH,} \mathrm{and} \mathrm{uptake}$ of $0.1 \mathrm{mM}\left[{ }^{3} \mathrm{H}\right]$ taurocholate was assayed in equiosmolar Hepes-Tris buffer, $\mathrm{pH} 7.4$, containing $50 \mathrm{mM}$ potassium and $0.5 \mathrm{mM}$ PAH in the presence or absence of $50 \mu \mathrm{M}$ valinomycin.

$¥$ Values represent the mean \pm SE for two runs of triplicate determinations. Values are expressed as pmol $\cdot \mathrm{mg}$ protein $^{-1}$. either under PAH-transstimulated or non-PAH-transstimulated conditions.

The final experiment was performed to test the interaction of a variety of inorganic and organic anions with PAH-transstimulated bile acid transport, i.e. taurocholate-PAH exchange. Ileal basolateral membranes were preincubated in the presence or absence of $10 \mathrm{mM}$ PAH and then exposed to incubation buffer containing radiolabeled $0.1 \mathrm{mM}$ taurocholate and various test anions. Fig. 7 shows 20-s uptake of taurocholate expressed as a percent of equilibrium $(60 \mathrm{~min})$ uptake in the presence of a PAH gradient (open bars) or in the absence of PAH (hatched bars). A lack of variability was seen for equilibrium values between various test anions and in the presence and absence of $\mathrm{PAH}$, e.g., the mean $\pm \mathrm{SE}$ for all experimental determinations under PAH-transstimulated and no PAH conditions were $305.0 \pm 2.3(n=48)$ and $306.4 \pm 1.6(n=48) \mathrm{pmol} \cdot \mathrm{mg} \mathrm{protein}^{-1}$, respectively. The paired bars at the left side of Fig. 7 show that a PAH gradient resulted in a $126 \%$ increase in initial taurocholate uptake over equilibrium uptake when compared with control vesicles containing no PAH. This PAH-transstimulated uptake of taurocholate was cis-inhibited significantly by $5 \mathrm{mM}$ pyruvate, $0.1 \mathrm{mM}$ taurocholate, $0.5 \mathrm{mM}$ cholate, $5 \mathrm{mM}$ PAH, $5 \mathrm{mM}$ chloride, $5 \mathrm{mM}$ sulfate, and $5 \mathrm{mM}$ bicarbonate to values approaching those seen for uptake in the absence of PAH. Taurocholate uptake in the absence of PAH was unaffected by the presence of these test anions.

\section{Discussion}

These studies directly examine for the first time bile acid transport in a highly enriched, intestinal basolateral membrane preparation. Taurocholate is an amphipathic substance that is capable

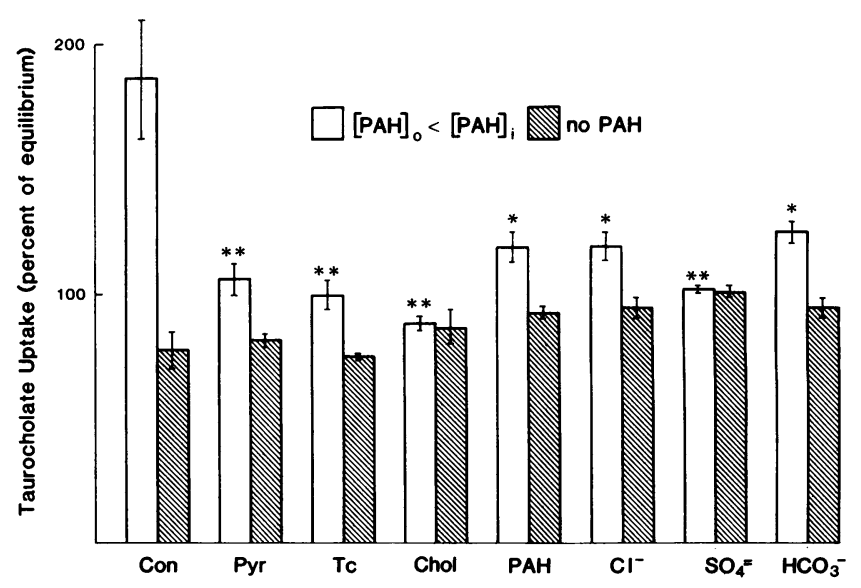

Figure 7. Effect of cis-inhibition on PAH-transstimulated taurocholate uptake by organic and inorganic anions. Ileal basolateral membrane vesicles were preloaded with $150 \mathrm{mM}$ tetramethylammonium, $50 \mathrm{mM}$ potassium, $200 \mathrm{mM}$ gluconate, $12 \mathrm{mM}$ Hepes-Tris, $\mathrm{pH} \mathrm{7.4,} \mathrm{and} 0$ (hatched bars) or $10 \mathrm{mM}$ PAH and $190 \mathrm{mM}$ gluconate (open bars). Vesicles then were incubated in the same PAH-free medium containing $0.1 \mathrm{mM}\left[{ }^{3} \mathrm{H}\right]$ taurocholate and either $5 \mathrm{mM}$ gluconate (control [Con]), $5 \mathrm{mM}$ pyruvate (Pyr), $1 \mathrm{mM}$ taurocholate (Tc), $0.5 \mathrm{mM}$ cholate (Chol), $5 \mathrm{mM}$ PAH, $5 \mathrm{mM}$ chloride, $5 \mathrm{mM}$ sulfate, or $5 \mathrm{mM}$ bicarbonate. All experiments were performed in the presence of valinomycin and FCCP. 20-s taurocholate uptake is expressed as percent of 60 -min (equilibrium) uptake. Each point represents the mean $\pm \mathrm{SE}$ for two runs of triplicate determinations. *Equals $P<0.05$, and ${ }^{* *}$ equals $P<0.01$ for PAH-driven uptake of control vs. other values. 
of partitioning between the aqueous phase and membrane lipid (27). Therefore, initial studies were performed to differentiate binding to the membrane from transmembrane transport. Evidence for transmembrane transport was obtained from osmolarity dependence and transstimulation experiments. An analysis of the osmolarity experiments indicated that only $30 \%$ of the taurocholate uptake at equilibrium could be accounted for by an osmotically-sensitive internal space. It is assumed that this part represents free taurocholate kept in the intravesicular volume. Washing experiments with unlabeled taurocholate suggested that $15 \%$ of taurocholate may be bound to the outer surface of the vesicles. The remaining taurocholate may have partitioned into the membrane interior and bound to the inner membrane surface $(23,24,28)$. Membrane translocation rather than merely binding is suggested further by the transstimulation of bile acid by other anionic species. Initial rates of taurocholate uptake were enhanced by externally directed anion gradients as opposed to gradient-free conditions. Transport rather than binding explains the transient intravesicular accumulation of taurocholate above equilibrium concentrations when anion gradient conditions were imposed across the vesicle membrane.

Since an anion exchange system has been described in the renal and intestinal basolateral membranes $(15,25,26)$, it seemed reasonable to determine whether such a transport system in the intestinal basolateral membrane was shared by the anionic bile acids. Like for the renal membranes (15), sulfate uptake by the basolateral membranes from jejunum and ileum was stimulated by a $\mathrm{pH}$ difference. Moreover, this proton gradient-driven sulfate transport was inhibited by taurocholate. Both sulfate and taurocholate uptake was transstimulated by bicarbonate, and bicarbonate-stimulated sulfate uptake was inhibited by taurocholate. Finally, sulfate transstimulated taurocholate uptake. In as much as these data suggested that taurocholate interacted with sulfate transport, other previously described characteristics of the sulfate anion exchange in renal basolateral membrane vesicles were examined for taurocholate transport by ileal basolateral membrane vesicles. Taurocholate uptake was transstimulated by a PAH gradient. The transstimulation of taurocholate uptake was cis-inhibited by pyruvate, PAH, probenecid, chloride, sulfate, and bicarbonate, as well as by taurocholate and cholate. Thus, representative anions that showed a transstimulation also cisinhibited taurocholate uptake.

The stimulation of sulfate uptake by a proton gradient and the cis-inhibition and transstimulation by various anions of sulfate or taurocholate uptake can be ascribed either to direct interaction with a common anion transport system or to indirect effects. These indirect effects arise from the coupling of radiolabeled substrate uptake to transmembrane electrical potential and/or pH differences that are generated by test anions (15). To minimize this possibility, experiments were performed under voltage and $\mathrm{pH}$ clamp conditions, i.e., in the presence of valinomycin, FCCP, and equimolar extra- and intravesicular potassium concentrations. Direct proof for complete clamping of transmembrane potential difference under all conditions is lacking because a co-transport system that is well recognized to be sensitive to changes in membrane potential is not present in intestinal basolateral membrane vesicles. However, FCCP, valinomycin and equimolar potassium concentrations inhibited the $\mathrm{H}^{+}$-gradient-driven sulfate uptake (Fig. 3). Therefore, it is unlikely that anion gradients stimulated sulfate and taurocholate uptake by changes in transmembrane $\mathrm{pH}$. Moreover, whether the voltage clamp was effective or not may not matter, as trans- stimulation of taurocholate by $\mathrm{PAH}$, at least, is insensitive to a transmembrane potential (Table I).

The interposition of a $\mathrm{NaCl}$ gradient (out greater than in) failed to stimulate taurocholate uptake. The substitution of a $\mathrm{NaCl}$ gradient by $\mathrm{Na}$ gluconate also did not result in stimulation of taurocholate uptake (data not shown). Thus, it is unlikely that a failure to demonstrate a sodium gradient stimulation can be explained by a simultaneous chloride cis-inhibition. Moreover, the absence of a sodium-stimulated taurocholate uptake suggests that our basolateral membrane preparation was not contaminated by brush-border membrane vesicles that contain a sodium-taurocholate co-transport system (9-12). The present findings appear consistent with the sodium-independent uptake of taurocholate by canalicular membrane vesicles of the rat hepatocyte (24). Observations with plasma membrane preparations from intestine, liver, and kidney suggest a mechanism for the vectorial transport of bile acids. Uptake across the brush-border membrane of ileum (9-12) and kidney (29) and sinusoidal membrane of the hepatocyte (23) occurs predominantly by a sodium-dependent transport system that is driven by the transmembrane sodium gradient. At the basolateral membrane of the ileum and canalicular membrane of the liver, bile acid leaves the cell by a sodium-independent transport system which, in the small intestine, can occur by anion exchange.

\section{Acknowledgments}

This work was supported by grant AM32045 from the National Institutes of Health.

\section{References}

1. Krag, E., and S. F. Phillips. 1974. Active and passive bile acid absorption in man. J. Clin. Invest. 53:1686-1694.

2. Schiff, E. R., N. C. Small, and J. M. Dietschy. 1972. Characterization of the kinetics of the passive and active transport mechanisms for bile acid absorption in the small intestine and colon of the rat. $J$. Clin. Invest. 51:1351-1362.

3. Dietschy, J. M., H. S. Solomon, and M. D. Siperstein. 1966. Bile acid metabolism. I. Studies on the mechanisms of intestinal transport. J. Clin. Invest. 45:832-846.

4. Holt, P. R. 1964. Intestinal absorption of bile salts in the rat. Am. J. Physiol. 207:1-7.

5. Lack, L., and I. M. Weiner. 1966. Intestinal bile salt transport: structure-activity relationships and other properties. Am. J. Physiol. 210: $1142-1152$.

6. Wilson, F. A., and J. M. Dietschy. 1972. Characterization of bile acid absorption across the unstirred water layer and brush border of the rat jejunum. J. Clin. Invest. 51:3015-3025.

7. Wilson, F. A., and L. L. Treanor. 1975. Characterization of the passive and active transport mechanisms for bile acid uptake into rat isolated intestinal epithelial cells. Biochim. Biophys. Acta. 406:280-293.

8. Wilson, F. A., and L. L. Treanor. 1981. Studies of relationships among bile acid uptake, $\mathrm{Na}^{+}, \mathrm{K}^{+}$-ATPase, and $\mathrm{Na}^{+}$gradient in isolated cells from rat ileum. Gastroenterology. 81:54-60.

9. Lücke, H. G., G. Stange, R. Kinne, and H. Murer. 1978. Taurocholate sodium co-transport by brush border membrane vesicles isolated from rat ileum. Biochem. J. 174:951-958.

10. Beesley, R. C., and R. G. Faust. 1979. Sodium ion-coupled uptake of taurocholate by intestinal brush border membrane vesicles. Biochem. J. 178:299-303.

11. Lack, L., J. T. Walker, and C. H. Hsu. 1977. Taurocholate uptake by membrane vesicles prepared from ileal brush borders. Life Sci. 20: 1607-1612.

12. Wilson, F. A., and L. L. Treanor. 1979. Glycodeoxycholate 
transport in brush border membrane vesicles isolated from rat jejunum and ileum. Biochim. Biophys. Acta. 554:430-440.

13. Wilson, F. A. 1981. Intestinal transport of bile acids. Am. J. Physiol. 241:G83-G92.

14. Wright, E. M., C. H. VanOs, and A. K. Mircheff. 1980. Sugar uptake by intestinal basolateral membrane vesicles. Biochim. Biophys. Acta. 597:112-124.

15. Low, I., T. Friedrich, and G. Burckhardt. 1984. Properties of an anion exchanger in rat renal basolateral membrane vesicles. Am. J. Physiol. 246:F334-F342.

16. Small, D. M. 1971. The physical chemistry of cholanic acid. In The Bile Acids. P. P. Nair and D. Kritchevsky, editors. Plenum Press, New York. 1:249-356.

17. Scalera, V., C. Storelli, C. Storelli-Joss, W. Haase, and H. Murer. 1980. A simple and fast method for the isolation of basolateral plasma membranes from rat small-intestinal epithelial cells. Biochem. J. 186: 177-181.

18. Scharschmidt, B. F., E. B. Keeffee, N. M. Blankenship, and R. K. Ockner. 1979. Validation of a recording spectrophotometric method for measurement of membrane-associated $\mathrm{Mg}$ - and NaK-ATPase activity. J. Lab. Clin. Med. 93:790-799.

19. Sottocasa, G. L., B. Kaylenstierna, L. Ernster, and A. Bergstrand. 1967. An electron-transport system associated with the outer membrane of liver mitochondria. J. Cell Biol. 32:415-438.

20. Fleischer, S., and B. Fleischer. 1967. Removal and binding of polar lipids in mitochondria and other membrane systems. Methods Enzymol. 10:406-432.

21. Lowry, O. H., N. J. Rosebrough, A. L. Farr, and R. J. Randall.
1951. Protein measurement with the folin phenol reagent. J. Biol. Chem. 193:265-275.

22. Hopfer, U., K. Nelson, J. Perrotto, and K. J. Isselbacher. 1973. Glucose transport in isolated brush border membrane from rat small intestine. J. Biol. Chem. 248:25-32.

23. Inoue, M., R. Kinne, T. Tran, and I. M. Arias. 1982. Taurocholate transport by rat liver sinusoidal membrane vesicles: evidence of sodium co-transport. Hepatology. 2:572-579.

24. Inoue, M., R. Kinne, T. Tran, and I. M. Arias. 1984. Taurocholate transport by rat liver canalicular membrane vesicles: evidence for the presence of an $\mathrm{Na}^{+}$-independent transport system. J. Clin. Invest. 73: 659-663.

25. Pritchard, J. B., and J. L. Renfro. 1983. Renal sulfate transport at the basolateral membrane is mediated by anion exchange. Proc. Natl. Acad. Sci. USA. 80:2603-2607.

26. Hagenbuch, B., G. Stange, and H. Murer. 1985. Transport of sulphate in rat jejunal and rat proximal tubular basolateral membrane vesicles. Pflügers Arch. Eur. J. Physiol. 405:202-208.

27. Wilson, F. A., and L. L. Treanor. 1977. Characterization of bile acid binding to rat intestinal brush border membranes. J. Membr. Biol. 33:213-230.

28. Hildman, B., C. Storrelli, W. Haase, M. Barac-Nieto, and H. Murer. 1980. $\mathrm{Na}^{+}$-L-Lactate cotransport in rabbit small intestinal brush border membrane vesicles. Biochem. J. 186:169-176.

29. Wilson, F. A., G. Burckhardt, H. Murer, G. Rumrich, and K. J. Ullrich. 1981. Sodium-coupled taurocholate transport in the proximal convolution of the rat kidney in vivo and in vitro. J. Clin. Invest. 67: 1141-1150. 